\title{
Experiencia en embolización supraselectiva de arterias vesicales para manejo de hematuria incoercible
}

\author{
Palma Ceppi C*, Reyes Osorio D*, Palma Ceppi R***, Palavecino P**. \\ *Servicio de Urología. ${ }^{* *}$ Médico cirujano. ${ }^{* * *}$ Radiologo. Hospital Clínico. Universidad de Chile. \\ Actas Urol Esp. 2008;32(5):542-545
}

\begin{abstract}
RESUMEN
EXPERIENCIA EN EMBOLIZACIÓN SUPRASELECTIVA DE ARTERIAS VESICALES PARA MANEJO DE HEMATURIA INCOERCIBLE

Introducción: La hematuria, uno de los principales motivos de consulta urológica, es producida por un amplio número de patologías desde infecciones urinarias hasta neoplasias malignas. El manejo de la hematuria incoercible puede requerir la acción de Radiología Intervencional en aquellos casos en que la patología queda fuera del alcance terapéutico habitual. Esta es una aproximación menos invasiva para el tratamiento de esta patología.

Material y métodos: Revisión retrospectiva de 6 casos de embolización supraselectiva de arterias vesicales con micropartículas, en hematurias macroscópica incoercible. Seguimiento a corto y mediano plazo.

Resultado: Cuatro pacientes tuvieron una evolución favorable, con desaparición de la hematuria macroscópica a las 48 horas del procedimiento. En dos casos se requirió de un segundo procedimiento endovascular (coils) a una semana. Los pacientes no presentaron complicaciones relacionadas a la intervención, durante el período de seguimiento.

Discusión: En pacientes no aptos para resolución quirúrgica de hematuria o en aquellos con falla de tratamiento habitual, la embolización supraselectiva parece ser una alternativa viable y sin complicaciones inmediatas. Se requieren estudios con seguimiento más prolongado y mayor número de casos para probar esta hipótesis.

Palabras clave: Hematuria. Embolización. Embolización selectiva. Embolización percutánea. Vesical. Vejiga.
\end{abstract}

\section{ABSTRACT \\ EXPERIENCE IN SUPERSELECTIVE EMBOLIZATION OF BLADDER ARTERIES IN THE TREATMENT OF INTRACTABLE HEMATURIA}

Introduction: The hematuria, one of the main motives of urologic consultations, is produced by an extensive number of urinary pathologies from infections to cancer. The management of hematuria can require the action of Interventional Radiology in those cases in which the pathology is outside of surgery therapy. This is an approximation less invasive for the management of this pathology.

Material and Methods: Retrospective review of 6 cases of supraselective embolization of bladder arteries with micropaticles in intractable macroscopic hematuria with associated anemia. Short and extensive monitoring.

Results: Four patients had a favorable evolution, with disappearance of macroscopic hematuria at 48 hours of the procedure. In two cases it was necessary a second endovascular procedure (coils) one week later. The patients did not present complications related to the intervention during the period of current monitoring.

Conclusion: In ineligible patients for surgical resolution of hematuria or in those with failure of habitual processing, the selective embolization seems to be a viable alternative and without immediate complications for the management. Studies with greater number of cases and more prolonged monitoring are required to test this hypothesis.

Keywords: Hematuria. Haemorrhage. Embolization. Selective embolization. Percutaneous embolization. Vesical. Bladder. 
$\mathrm{L}^{\mathrm{a}}$ a hematuria incoercible de origen vesical es un cuadro clínico de difícil manejo y que puede comprometer la vida del paciente. Múltiples son las patologías que pueden ocasionarlo, como cáncer vesical, cistitis actínica, cistitis hemorrágica secundaria a quimioterápicos o, menos frecuentemente, infecciones urinarias ${ }^{1}$. La mayoría de estos cuadros se presentan en pacientes añosos o en tratamiento paliativo de patologías oncológicas pelvianas, en los cuales se dificulta un manejo quirúrgico agresivo, siendo de elección el manejo conservador con terapias poco invasivas. Por otra parte, el tratamiento de pacientes que rechazan la transfusión de sangre y hemoderivados es otro grupo que ante hematuria incoercible requieren un rápido abordaje para detener el sangrado.

Múltiples son las terapias que se han descrito para el manejo de la hematuria, que van desde la irrigación vesical continua con solución fisiológica, irrigación vesical con alumbre o formalina, hidrodistensión vesical, y manejo específico de algunos casos como el uso de cámara hiperbárica en cistitis actínica $^{1}$. Dentro de estas terapias aparece la embolización arterial del flujo sanguíneo vesical como un tratamiento poco invasivo y con buenos resultados. La gran mayoría de la experiencia publicada con esta técnica consiste en la embolización de las arterias iliacas internas o sus ramas anteriores. Presentamos una serie de 6 casos de embolización supraselectiva de arterias vesicales en el manejo de la hematuria incoercible.

\section{MATERIAL Y MÉTODOS}

6 pacientes entre enero del 2003 y enero del 2007, con cuadros de hematuria incoercible y que no responden a tratamiento conservador, fueron sometidos a embolización supraselectiva de arterias vesicales. La edad promedio de los pacientes es de 66 años (rango entre 59 y 74 años). 4 pacientes de sexo masculino y 2 femenino. La etiología del sangrado fue cistitis actínica en 5 casos y cáncer vesical fuera de alcance quirúrgico en un caso. La paciente de sexo femenino además rechazaba transfusión de sangre y hemoderivados por motivos religiosos (Tabla 1).

\section{TÉCNICA}

Ubicación del sitio de sangrado y anatomía del sector con angiografía (Fig. 1). Ingreso por vía femoral bajo anestesia local para realizar cateterización selectiva o supraselectiva de los vasos arteriales que suplen el sitio de sangrado con catéter 2,5 Fr. Se utiliza material microparticulado, alcohol polivinílico de 500 a 700 micrones, para ocluir vasos de tamaño intermedio (Fig. 2), para disminuir en forma importante el aporte sanguíneo, sin someter al órgano a una isquemia severa que pudiese provocar necrosis. Posterior al procedimiento, los pacientes mantienen catéter vesical permanente hasta 24 horas de resuelto el sangrado.

\section{RESULTADOS}

A los 6 pacientes se les realizó embolización supraselectiva de arterias vesicales. Todos presen-

Tabla 1

\begin{tabular}{|c|c|c|c|c|c|c|}
\hline Paciente & 1 & 2 & $3 *$ & 4 & 5 & 6 \\
\hline Edad & 66 & 65 & 59 & 74 & 67 & 61 \\
\hline Sexo & Masculino & Masculino & Femenino & Masculino & Masculino & Femenino \\
\hline Patología & CP, PRR, RT, CA & $\mathrm{CV}$ & CCU, RT, CA & $\mathrm{CV}, \mathrm{RT}, \mathrm{CA}$ & CP, RT, CA & $\mathrm{CCU}, \mathrm{RT}, \mathrm{CA}$ \\
\hline Tratamiento Inicial & $\begin{array}{l}\text { Irrigación con } \\
\text { alumbre, IVC }\end{array}$ & IVC & IVC & IVC & IVC & $\begin{array}{l}\text { Irrigación } \\
\text { con alumbre, } \\
\text { IVC }\end{array}$ \\
\hline Resultado & $\begin{array}{l}\text { Control } \\
\text { primer } \\
\text { intento }\end{array}$ & $\begin{array}{l}\text { Control } \\
\text { primer } \\
\text { intento }\end{array}$ & $\begin{array}{l}\text { Control } \\
\text { segundo } \\
\text { intento }\end{array}$ & $\begin{array}{l}\text { Control } \\
\text { segundo } \\
\text { intento }\end{array}$ & $\begin{array}{l}\text { Control } \\
\text { primer } \\
\text { intento }\end{array}$ & $\begin{array}{l}\text { Control } \\
\text { primer } \\
\text { intento }\end{array}$ \\
\hline $\begin{array}{l}\text { Seguimiento } \\
\text { (meses) }\end{array}$ & 16 & 6 & 14 & 9 & 8 & 1 \\
\hline
\end{tabular}

\footnotetext{
* Paciente rechaza transfusión de sangre y hemoderivados por motivos religiosos

$\mathrm{CP}=$ Cáncer de Próstata $\quad \mathrm{PRR}=$ Prostatectomía Radical Retropúbica

$\mathrm{RT}=$ Radioterapia

$\mathrm{CA}=$ Cistitis Actínica

$\mathrm{CV}=$ Cáncer de Vejiga

$\mathrm{CCU}=$ Cáncer Cervicouterino

IVC = Irrigación Vesical Continua
} 


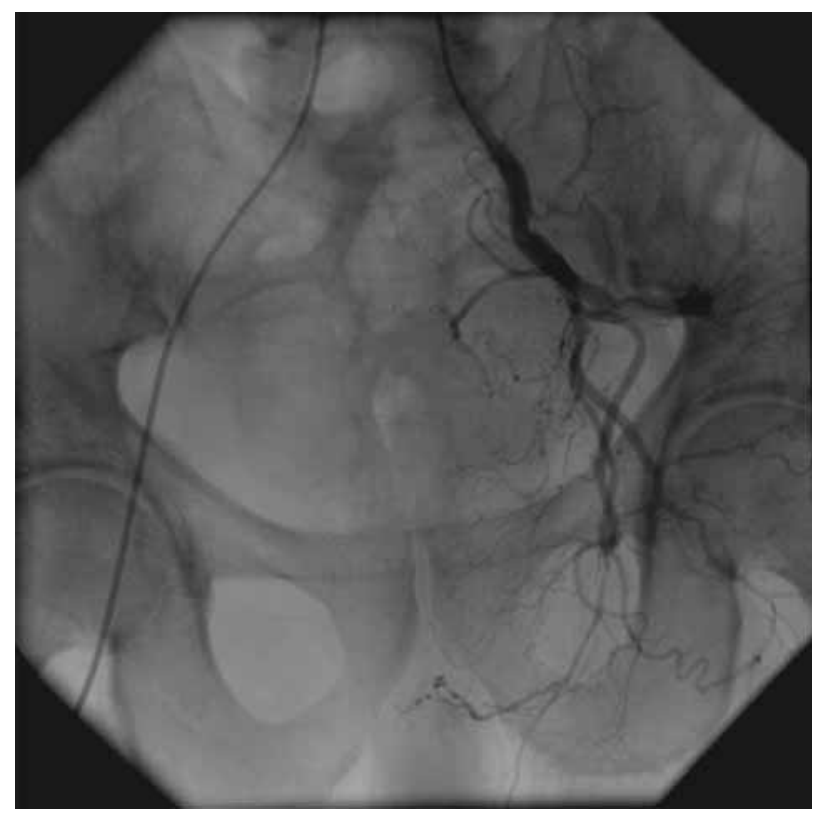

FIGURA 1. Angiografía previa a embolización.

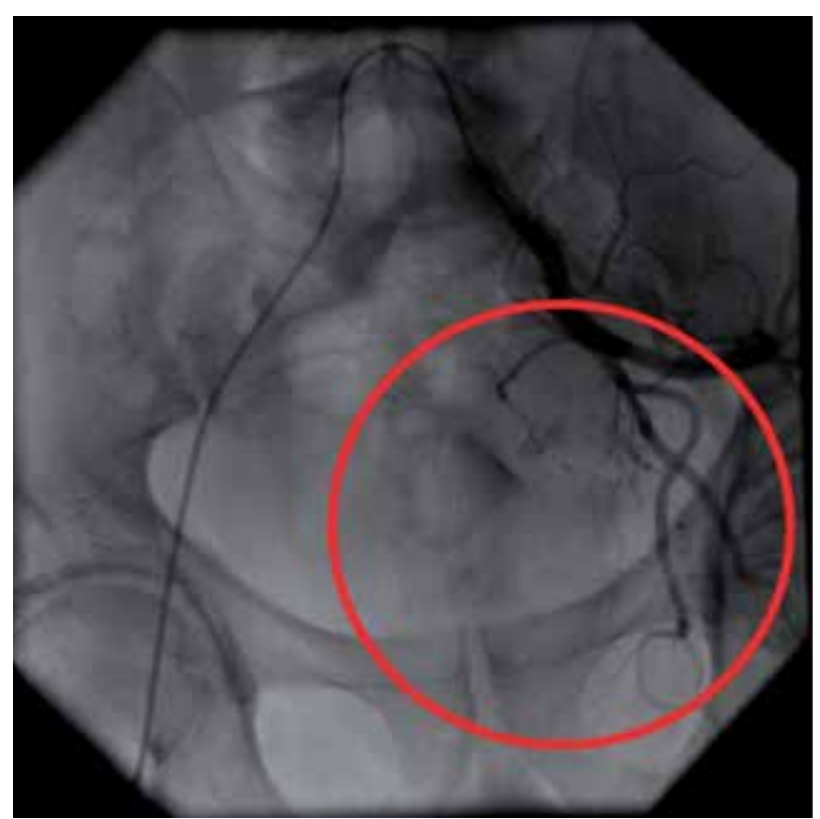

FIGURA 2. Imagen posterior a embolización

taron remisión de la hematuria a las 48 horas. 2 pacientes presentaron recidiva del sangrado durante la primera semana posterior al procedimiento, por lo que requirieron reintervención: un paciente fue sometido a una nueva embolización supraselectiva de arterias vesicales con material microparticulado, y al otro paciente se le instaló un coil de $4 \mathrm{~mm}$ en la rama anterior de la arteria iliaca interna izquierda. Ambos pacientes respondieron al tratamiento, con desaparición de hematuria a las 48 horas de los procedimientos.

El seguimiento post egreso hospitalario, con un tiempo promedio de 9 meses (rango entre 1 y 16 meses) no mostró recidiva de la hematuria en ningún caso. En nuestra serie no existieron complicaciones intra ni postoperatorias.

\section{DISCUSIÓN}

El uso de la embolización en el manejo de la hematuria incoercible ha sido descrito desde hace $30 \mathrm{año}^{2}$. En el sangrado de origen vesical, la mayoría de los casos cede al tratamiento con irrigación vesical o con manejo endoscópico. En 1974, Hald describió el primer procedimiento de embolización para hematuria incoercible de origen vesical, con la embolización de arterias ilíacas internas o sus ramas anteriores ${ }^{2-5}$, con resultados satisfactorios en el $92 \%$ de los casos $^{6}$. Se han descrito técnicas más selectivas de emobilización, como la embolización de arterias vesicales ${ }^{7,8}$ o de ramas de éstas que irrigan la zona de sangrado. Ésta última técnica fue publicada por primera vez por De Berardinis et $\mathrm{al}^{9}$ ), presentando un caso de hematuria incoercible por cistitis actínica, con cese del sangrado posterior al procedimiento y sin complicaciones intra y postoperatorias.

En nuestra serie, la embolización supraselectiva de las ramas de arterias vesicales que irrigan el sitio de sangrado ha presentado buenos resultados, con una respuesta completa e inmediata en 4 pacientes, sin recidiva a 9 meses promedio. Nuestra serie es la primera presentada desde que De Berardinis publicó el primer caso ${ }^{9}$.

Se han utilizado distintos materiales para la embolización, como músculo ${ }^{2}$, coágulos antólogos ${ }^{1}$ y gelatinas ${ }^{10}$. Nosotros utilizamos material microparticulado de alcohol polivinílico, de 500 a 700 micrones, que permite una buena hemostasia, sin oclusión total del flujo sanguíneo, para disminuir la posibilidad de isquemia.

Las complicaciones descritas por la embolización pueden ser graves, y han sido atribuidas a técnicas poco selectivas como la embolización las arterias iliacas internas, aunque una serie reciente no ha mostrado complicaciones ${ }^{5}$. La complicación más frecuente es dolor glúteo por oclusión de la arteria glútea $^{11}$ ). También se ha descrito la isquemia de 
extremidades inferiores por émbolos ${ }^{10}$, gangrena vesical $^{12,13}$ y una complicación rara como la paraplejia o plejia de una extremidad inferior, probablemente por embolización de arterias espinales y compromiso medular secundario ${ }^{14}$. Las técnicas más selectivas no han presentado complicaciones, similar a lo ocurrido con nuestros pacientes.

En conclusión, la embolización supraselectiva de arterias vesicales es una técnica útil en el manejo de la hematuria incoercible, sobretodo en pacientes sin opción quirúrgica, con buenos resultados y sin complicaciones.

\section{REFERENCIAS}

1. Choong SK, Walkden M, Kirby R. The management of intractable haematuria. BJU Int. 2000;86(9):951-959.

2. Hald T, Myging T. Control of life-threatening vesical hemorrhage by unilateral hypogastric artery muscle embolization. J Urol. 1974;112(1):60-63.

3. Carmignani G, Belgrano E, Puppo P, Cichero A, Giuliani L. Transcatheter embilization of the hypogastric arteries in cases of bladder hemorrhage from advanced pelvic cancers: followup in 9 cases. J Urol. 1980;124(2):196-200.

4. Jenkins CN, McIvor J. Survival after embolization of the internal iliac arteries in ten patients with severe haematuria due to recurrent pelvic carcinoma. Clin Radiol. 1996;51(12):865-868.

5. Nabi G, Sheikh N, Greene D, Marsh R. Therapeutic transcatheter arterial embolization in the management of intractable haemorrhage from pelvic urological malignancies: preliminary experience and long-term follow-up. BJU Int. 2003;92(3):245-247.

6. McIvor J, Williams G, Southcott RD. Control of severe vesical haemorrhage by therapeutic embolisation. Clin Radiol. 1982;33 (5):561-567.
7. Kobayashi T, Kusano S, Matsubayashi T, Uchida T. Selective embolization of the vesical artery in the management of massive bladder hemorrhage. Radiology. 1980;136(2):345-348.

8. Palandri F, Bonifazi F, Rossi C, Falcioni S, Arpinati M, Giannini $\mathrm{MB}$, et al. Successful treatment of severe hemorrhagic cystitis with selective vesical artery embolization. Bone Marrow Transplant 2005;35(5):529-530.

9. De Berardinis E, Vicini P, Salvatori F, Sciarra A, Gentile V, Di Silverio F. Superselective embolization of bladder arteries in the treatment of intractable bladder haemorrhage. Int $\mathrm{J}$ Urol. 2005; 12(5):503-505.

10. Giuliani L, Carmignani E, Belgrano E, Puppo P. Gelatin foam and isobutyl-2-cyanoacrylate in the treatment of life-threatening bladder haemorrhage by selective transcatheter embolisation of the internal iliac arteries. Br J Urol. 1979;51(2):125128.

11. Greenstein A, Merimsky E, Papo J, Braf Z. Persistent gluteal pain after embolization of the hypogastic arteries. An unexpected complication. J Urol (Paris) 1983;89(8):595-596.

12. Braf ZF, Koontz WW. Gangrene of the bladder. Complication of hypogastric artery embolization. Urology. 1977;9(6):670-671.

13. Hietala SO. Urinary bladder necrosis following selective embolization of the internal iliac artery. Acta Radiol Diagnosis (Stockh). 1978;19(2):316-320.

14. Hare WS, Holland CJ. Paresis following internal iliac artery embolisation. Radiology. 1983;146(1):47-51.

Correspondencia autor: Dr. C.A. Palma Ceppi Servicio de Urología. Hospital Clínico.

Universidad de Chile. Avda. Santos Dumont, 999

8380456 Independencia. Santiago. Chile

E-mail autor: palmaceppi@gmail.com

Información artículo: Original - Urología general

Trabajo recibido: junio 2007

Trabajo aceptado: julio 2007 